\title{
Studying Molecular Aspects of the Blood-Brain Barrier Using an in Vitro Model: Contribution of a Global Proteomics Strategy
}

\author{
Yannis Karamanos \\ Laboratoire de la barrière hémato-encéphalique, Université d’Artois, Lens, France \\ Email: yannis.karamanos@univ-artois.fr
}

Received October 2013

\begin{abstract}
A global proteomics strategy was initiated to decipher molecular mechanisms associated with the blood-brain barrier (BBB) phenotype of the brain capillary endothelial cells. The different methods implemented were shown complementarily. The main results obtained using an in vitro BBB model allowed highlighting the role of several protein actors of cytoskeleton remodelling, the involvement of the asymmetric dimethylarginine pathway in regulating endothelial function and the role of tissue non-specific alkaline phosphatase in the regulation of endothelial permeability.
\end{abstract}

\section{Keywords}

Blood-Brain Barrier; BBB; Endothelial Cells; Proteomics

\section{Introduction}

The blood-brain barrier (BBB) corresponding to the cerebral capillaries covers approximately $95 \%$ of the total area of barriers between blood and brain [1]. The morphology and functional properties of the brain capillary endothelial cells (BCEC) that form with other cells (Figure 1) the BBB are well documented: a decrease in endothelial permeability, fewer caveolae, the reinforcement of tight junctions, fewer pinocytic vesicles, an increase in the number of mitochondria and a higher transendothelial electrical resistance [2,3]. The BBB contributes to the brain homeostasis by controlling the passage of endogenous and exogenous compounds and this is due, at least in part, to well-known proteins namely tight junction proteins, membrane transporters and metabolic barrier proteins. Closely associated to perivascular neurons, pericytes, and astrocytes, the BCEC constitute a functional neurovascular unit [4].

Since its discovery one hundred years ago, the BBB has been the centre of many studies and, in more recent years, it has been increasingly analyzed by "omics". The genomic and proteomic investigations carried out in order to enhance our understanding of the BBB were recently reviewed [5]. Primarily, this work has demonstrated that expression profiling technologies are powerful and provide significant information on the brain microvessel endothelium. Genomics and proteomics provide different complementary data on a tissue; genomics show relative expression of membrane and released proteins, while proteomics indicate protein modifications 


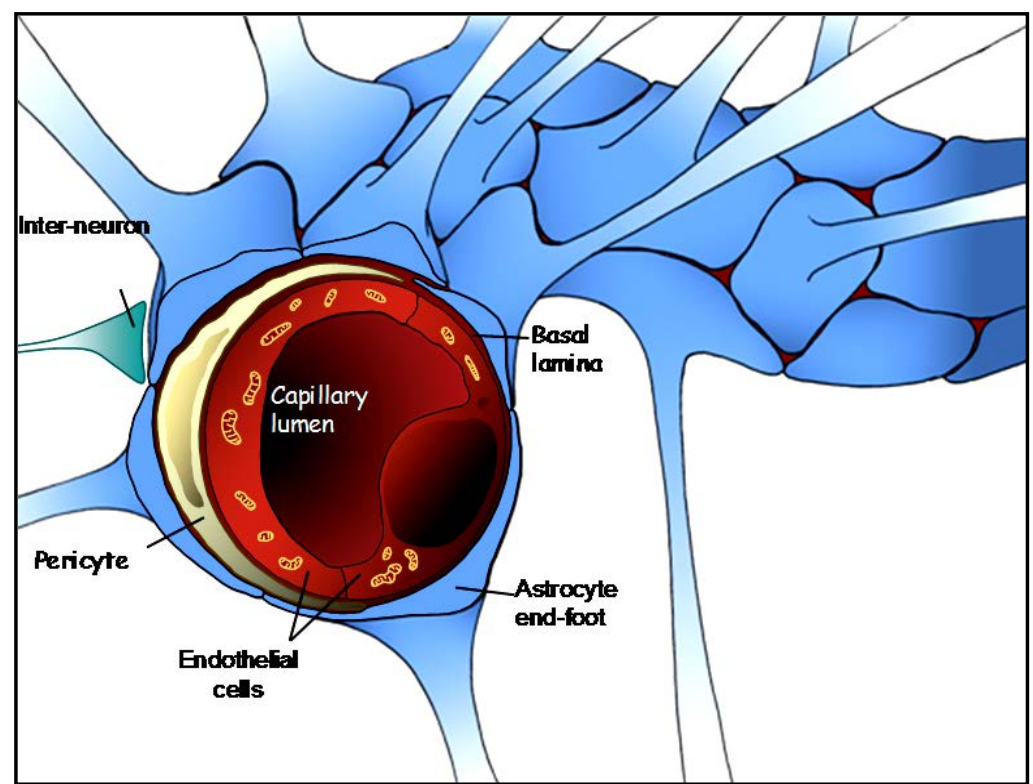

Figure 1. The endothelial cells are surrounded by a tubular sheath of astrocyte end-feet. Pericytes are embedded in the basal lamina (between the endothelium and the astrocyte end-feet). Reprinted from [5] with permission.

and isoforme expression. Moreover, the characterization of endothelial cells highlights some brain specific proteins which could explain BBB features.

Study the BBB characteristics and features requires both in vivo and in vitro models each with unique advantages and disadvantages. Hence, in vivo experiments require high numbers of animals in order to isolate the microvasculature and the methods used could provoke stress for the endothelial cells [5]. In contrast, the tissue complexity cannot be reproduced with in vitro models, but in turn those models are more flexible, the medium can be renewed and complemented and also large quantities of well characterised cells can be easily obtained. Consequently, the latter characteristic has formed the basis of the proteomics strategy described henceforth.

The in vitro BBB model developed in our laboratory utilizes bovine BCEC that were purified to homogeneity by mechanical homogenisation and filtration [6] and subsequent co-culturing with murine glial cells. These particular culture conditions induce differentiation of endothelial cells which provide an in vivo BBB phenotype [7].

As we have recently reported [8], the advantage of global proteomics strategies is that no hypothesis is required, other than a measurable difference in one or more protein species between the samples. Global proteomics methods attempt to separate quantify and identify all the proteins from a given sample. The separation step can be carried out directly on proteins or on the set of peptides derived from the enzymatic digestion of the corresponding proteins.

In the effort to decipher molecular mechanisms for the establishment of the BBB, we have experimented with several methods and we demonstrated that the in-gel $[9,10]$ and the off-gel $[9,11,12]$ approaches were complementary.

\section{Optimisation of the Protocol}

The in vitro model developed in our laboratory has two specificities, firstly the use of a collagen matrix, secondly the co-culture of the BCEC through a filter with glial cells. The first step was to adapt the technique to the model and to optimise the protocol to this culture mode. In preliminary experiments the particularities of the system were taken into account, 1) the fragility of the cells, 2) the presence of the collagen matrix and 3) the presence of serum proteins in the medium. Because cell harvesting, cell lysis and protein extraction procedures are unavoidable inseparable components of any cellular proteomics approach, the reproducibility directly depends on the care taken to prepare the protein samples. This first step could affect all the subsequent steps and of course, the final results. 


\subsection{Cell Culture}

The BCEC were isolated and characterized as described [13]. Primary cultures of mixed glial cells were made from newborn rat cerebral cortex [14]. Glial cells were cultured in DMEM supplemented with $10 \%(\mathrm{v} / \mathrm{v})$ heatinactivated foetal calf serum, $2 \mathrm{mM}$ glutamine and $50 \mu \mathrm{g} / \mathrm{ml}$ gentamycin. Three weeks after seeding, the glial cell cultures were stabilized and used for co-culture.

When isolated in vitro, primary bovine BCEC dedifferentiate and lose their BBB functionalities (limited BBB; Lim. BBB). The latter can be restored by 12 days of co-culture (Figure 2) with glial cells [6]. For each individual experiment, the re-induced BBB (Re-ind. BBB) properties were confirmed by 1) measuring an optimal value for the paracellular permeability coefficient (Pe) and 2) immunostaining the main tight junction proteins (ZO- 1 , occludin and claudin-5). Each time the markers display a pericellular distribution in differentiated BCEC, which contrasts with the uniform pattern for dedifferentiated cells $[11,15,16]$.

\subsection{Cell Harvesting}

In order to avoid contaminant proteins originating from the serum used in the culture media, several harvesting conditions were used. The BCEC were harvested after several washes with calcium/magnesium free-PBS (CMFPBS) according to a mechanical (scraped off into ice-cold CMF-PBS), a chemical (Versen TM solution) and an enzymatic method (porcine pancreas trypsin, or collagenase type XI) as described [10]. The enzymatic method using collagenase gave the best results [10,17], none of the highly-represented serum proteins such as BSA, heavy and light chains of IgG or a1-antitrypsin, were detected on the gel profiles.

\subsection{Protein Sample Preparation}

The cell pellet was subjected to a standardized ice-lysis procedure, by addition of $200 \mathrm{~mL}$ of lysis buffer (10 $\mathrm{mM}$ Tris-HCl, $1 \mathrm{mM}$ EDTA (Na), 1\% v/v Triton X-100, 0.1\% v/v 2-mercaptoethanol and protease inhibitors), with vigorous agitation for $5 \mathrm{~min}$ and sonication $(30 \mathrm{~W}, 20 \mathrm{~s})$. The remaining insoluble material was then pelleted by centrifugation $\left(13,500 \times \mathrm{g}, 4^{\circ} \mathrm{C}, 45 \mathrm{~min}\right)$ and stored at $-70^{\circ} \mathrm{C}$. Proteins in the supernatant were assayed with BSA as a standard [18]. The supernatant proteins were concentrated and delipidated overnight at $-20^{\circ} \mathrm{C}$ by addition of cold acetone (4 vol).

\section{Global Comparative Proteomics Approach}

Although marked progress has been made over the last decade, the process by which BCEC differentiate to obtain the BBB phenotype is poorly documented in molecular terms. In order to gain a deeper understanding of BBB-related molecular features, we have been applying a range of proteomic tools to our well-characterized in vitro BBB model for the last few years. We are particularly seeking to describe the protein abundance changes that occur during the glial cell-driven re-induction of the BBB phenotype in BCEC.

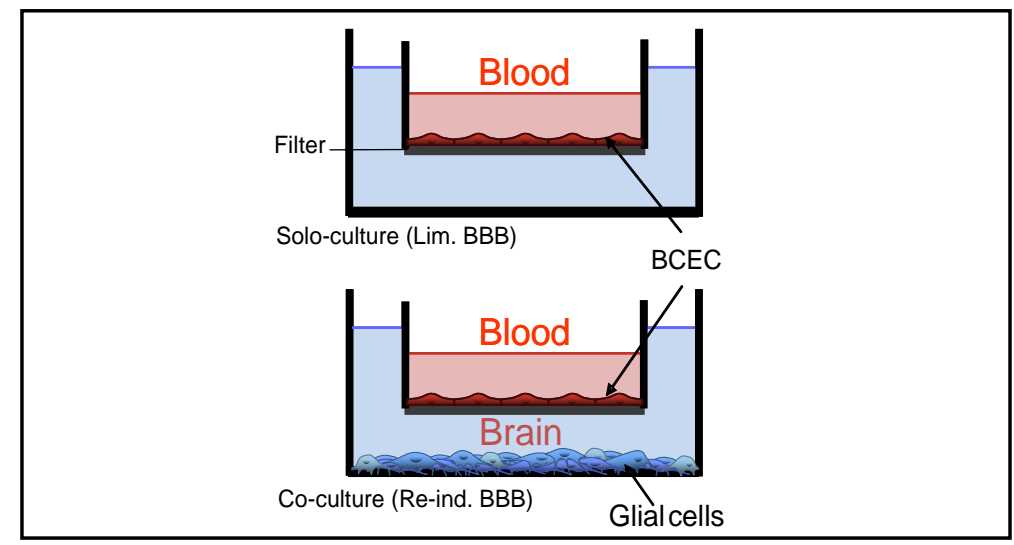

Figure 2. Schematic drawing of the culture system used in the in vitro BBB model, indicating the blood and brain compartments. Bovine BCEC and murine glial cells were used in this study. 


\subsection{Bioinformatics Resources and Protein Lists}

Protein lists were compared using nwCompare software [19]. All identified proteins were converted into gene names with the database for annotation, visualization and integrated discovery (DAVID) bioinformatics resources http://david.abcc.ncifcrf.gov/home.jsp [20], prior to sorting in the protein analysis through evolutionary relationships (PANTHER) classification system (www.pantherdb.org). Proteins are classified into families and subfamilies of shared function, which are then categorized by molecular function and biological process ontology terms [21,22].

\subsection{In-Gel Profiling}

The aim of the first approach was to determine by differential proteomic analysis the changes occurring in BCEC differentiated in co-culture with astrocytes compared with endothelial cells cultured alone. The two-dimensional gel electrophoresis (2-DE) steps, including the isoelectric focusing and SDS-PAGE conditions, the gel staining, the image acquisition and the comparative study were detailed elsewhere [17] together with examples of protein identification by peptide mass fingerprint (PMF) measured by MALDI-TOF-MS, and complementary data issued from peptide fragmentation fingerprints (PFF) that allow successful protein identifications. PMF- and PFF-analyses provide complementary datasets and thus, more comprehensive sequence coverage of the BCEC proteome, especially when they are combined together (PMF/PFF). Preliminary work [10] demonstrated the role of actin-binding and -bundling proteins, such as gelsolin, filamin-A, T-plastin and actin itself, in the establishment of the in vitro BBB phenotype of BCEC in response to stimulation by astrocytes. We also speculated that $\mathrm{Ca}^{2+}$ and PIP2 are involved in the response to this stimulation.

A thorough study [12] revealed differences in Triton-X-100-solubilized proteins from bovine BCECs with limited or re-induced BBB functionalities (cultured in the absence or presence of glial cells, respectively). The 81 proteins of differing abundance were linked to 55 distinct genes. According to the PANTHER classification system and Ingenuity Pathway Analysis, these quantitative changes mainly affected proteins involved in 1) cell structure and motility and 2) protein metabolism and modification processes. The fold-changes affecting HSPB1, moesin and ANXA5 protein levels were confirmed by western blot analysis but were not accompanied by changes in the corresponding mRNA expression levels. Our results revealed that the BCEC can adapt to variations in their environment and this involves the reorganization of the actin cytoskeleton contributing to the blood-brain barrier phenotype.

A differential gel electrophoresis (2D-DIGE)-based proteomics approach [23] confirmed that quantitative changes mainly concern proteins involved in cell structure and motility. Furthermore, the possible involvement of the asymmetric dimethylarginine (ADMA) pathway in the BBB phenotype re-induction process was suggested and potential role of ADMA in regulating endothelial function (in addition to its role as a by-product of protein modification) also anticipated. Purine nucleoside phosphorylase (PNPH) was also found to be more abundant in BCEC with Re.-ind. BBB phenotype. These results also suggested that the intracellular redox potential is lower in the in vitro brain capillary endothelial cells displaying re-induced BBB functions than in cells with limited BBB functions.

\subsection{Label-Free Off-Gel Profiling}

In this approach, the proteins in a sample are directly submitted to enzymatic digestion and the mixture of the genrated peptides, whose molecular mass ranges from 500 - $4000 \mathrm{Da}$, are separated and subsequently analysed by MS. A differential nano-LC MALDI-TOF/TOF-MS study was performed with Triton X-100-soluble protein species from bovine BCEC displaying either Lim. BBB or Re-ind. BBB functions [9]. The complexity of the crude extract of Triton X-100 solubilised proteins from BCEC prevented efficient mass spectrometry (MS) fragmentation analysis and, thus, the identification of individual proteins. To attenuate this phenomenon the extracts were fractionated into 5 fractions of increasing concentration in acetonitrile $(0 \%, 25 \%, 50 \%, 75 \%$ and $100 \%$ ) as previously described [11]. Each fraction was subjected to the off-line 1D-LC separation, in which high numbers of components were MS-detected, approximately $15 \%$ of them were in-source fragmented and most of the MS/MS spectra provided unambiguous protein identities in the main fractions (F0, F25, F50 and F75). Overall, the analysis enabled the identification of 436 and 408 proteins in bovine Lim. BBB and Re-ind. BBB, respectively. Eleven of these, ranging from proteins associated with assembly and organization of the cytoskele- 
ton [10] to those involved in vesicular transport and nucleic acid binding, appeared to be more abundantly in the cytoplasm of Re-ind. BBB cells, in particular tissue non-specific alkaline phosphatase (TNAP) and Eps15 homology domain-containing protein 1 (EDH1). This over-expression was accompanied by an increase in alkaline phosphatase (AP) enzymatic activity detected in the cells. Moreover, we found that endothelial permeability was significantly greater when AP activity was specifically inhibited with levamisole, suggesting that TNAP is involved in the regulation of endothelial permeability.

\subsection{Isotope-Coded Protein Label (ICPL) Profiling}

Besides the off-gel label-free profiling described above we started a quantitative evaluation of the differences in abundance between the BCEC with Lim. BBB and Re-ind. BBB functions via an ICPL approach, using a commercially available ICPL kit (Serva, Heidelberg, Germany). After their fractionation by increasing acetonitrile concentration, the proteins in each fraction (F0, F25, F50, F75) from Re-ind. BBB were labelled with the heavy isotope. Then, each fraction from Re-ind. BBB was mixed with the equivalent one from the Lim. BBB cells that were labelled with the light isotope. The consecutive nano-LC MALDI-TOF/TOF-MS analyses were performed in triplicate (unpublished data). Among the 412 proteins that were identified in at least two of the three replicates, 290 were quantified and when a threshold of 1.3 was retained for the regulation factor, 81 proteins were shown to be up-regulated in BCEC, with 34 and 47 in Re-ind. BBB and in Lim. BBB respectively.

\subsection{Proteome Reference}

The combination of 2-DE and 1D-LC approaches enabled the identification of about 430 Triton X-100-soluble proteins from BCEC with re-induced BBB functionalities [11]. Information on the proteins identified after 1D-LC MS/MS is shared through the PRIDE database for convenient comparison with proteomic datasets from non-brain vascular endothelial cells (http://www.ebi.ac.uk/pride under accession numbers 12825 to 12830). Due to the extraction conditions, only few proteins, less than $5 \%$, were membrane-associated proteins. More than $75 \%$ of the identified proteins display binding, catalytic or structural functions. Most identified proteins were involved in metabolic and cellular processes but transport and cell-cell communication process accounted for almost $25 \%$ of the identified species. This also emphasized the advantage of inter-species sequence homology comparisons for protein identification in non-completely sequenced genomes and highlighted the drifts and drawbacks generated by the rapid, gel-free proteomic methods, which nevertheless provide most of today's data. In the absence of a physiological, human in vitro BBB model, the proteomics expression profile of BCEC displaying BBB properties is the first step towards the creation of an anti-BBB antibody library which will greatly facilitate large-scale, array-based screening of protein expression.

\subsection{Membrane Proteins}

The distribution and the nature of plasma membrane (PM) proteins in BCEC was assessed after surface biotin labelling, isolation of the labelled proteins with streptavidin affinity chromatography and identification with nano-LC MS/MS. Very few cytoplasmic proteins, secreted proteins or proteins added to the cell culture medium were recovered-despite their relatively high cellular abundance. We reported [24] on the novel identification of transmembrane and membrane-associated proteins in bovine BCEC with re-induced BBB phenotype. Our findings demonstrated the efficiency of the enrichment approach used, even though only about 30 proteins came from the BCEC PM. The fact that transmembrane and membrane-associated proteins accounted for less than half the identified proteins showed how difficult it still is to isolate, solubilise and digest hydrophobic proteins of low cellular abundance. Our results suggest that the specific properties of PM proteins must be taken into account when seeking to improve biotinylation, purification and identification methods. Furthermore, this study reported the identification of several proteins involved in cellular endocytosis, membrane trafficking and receptor internalization (such as EHD2 and myoferlin) together with their cellular partners. These proteins and the pathways of which they are a part may become new targets for increasing drug transport across the BBB.

\section{Discussion}

The acquisition of the BBB phenotype of BCEC was largely studied at a physiological level but is far from being well understood at the molecular level. The composition and protein-protein relations at the plasma mem- 
Table 1. Identified proteins that were validated.

\begin{tabular}{|c|c|c|c|}
\hline \multirow{2}{*}{ Database entry name } & \multicolumn{3}{|c|}{ Proteins up-regulated or identified only in Lim. BBB or in Re-ind. BBB } \\
\hline & Protein name & Lim. BBB & Re. Ind BBB \\
\hline FLNA_HUMAN & Filamin-A & $\mathrm{X}$ & \\
\hline GELS_BOVIN & Gelsolin & & $\mathrm{X}$ \\
\hline PLST_HUMAN & Plastin-3 (T-plastin) & & $\mathrm{X}$ \\
\hline ACTB_CAMDR & Actin, cytoplasmic 1 & $\mathrm{X}$ & \\
\hline PNPH_BOVIN & Purine nucleoside phosphorylase & & $\mathrm{X}$ \\
\hline DDAH-1 & Dimethylarginine dimethylaminohydrolase & & $\mathrm{X}$ \\
\hline PPBT_BOVIN & Alkaline phosphatase, tissue non specific & & $\mathrm{X}$ \\
\hline EHD1_BOVIN & EH-domain containing protein 1 & & $\mathrm{X}$ \\
\hline
\end{tabular}

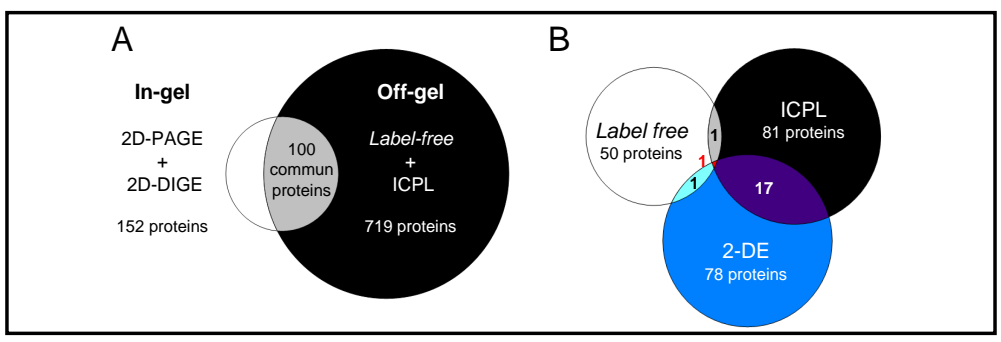

Figure 3. Label-free, ICPL and 2-DE approaches are complementary (A) Number of proteins identified by the in-gel and off-gel approaches (B) Comparison of the proteins identified, and showing quantitative variations, by three different ways.

brane level of BCEC are now well documented $[25,26]$ but the cell differentiation process remains curiously less known at the cytoplasmic level. The solubilisation properties of the Triton X-100 allowed us to investigate a sub-proteome mainly composed of cytoplasmic proteins and thus to try to reveal potential molecular mechanisms involved in the establishment of the BBB phenotype.

Using in-gel profiling (2-DE and 2D-DIGE) 152 different proteins were identified [10-12,23]. The off-gel profiling (label-free and ICPL) allowed the identification of 719 proteins with only 100 common proteins between the two approaches, thus demonstrating that they are complementary (Figure 3A). This fact is reinforced when comparing the proteins identified and showing quantitative variations by three different ways (Figure 3B). Among the 166 proteins (total for Label-free, ICPL and 2-DE put together), 19 were found in two approaches and only 1 in all three. The main proteins identified during the global proteomics approach presented above and that were subjected to RT-PCR and/or immunoblotting validation to confirm quantitative changes are presented in Table 1. The obtained results allowed highlighting the role of several protein actors of cytoskeleton remodelling, the involvement of the asymmetric dimethylarginine pathway in regulating endothelial function and the role of tissue non-specific alkaline phosphatase in the regulation of endothelial permeability. Furthermore, this study reported the identification of several proteins involved in cellular endocytosis, membrane trafficking and receptor internalization. Among the remaining identified proteins a selection is currently under investigation and will soon complete this figure.

\section{Acknowledgements}

I wish to particularly thank Gwënaël Pottier, Barbara Deracinois, Sophie Duban-Deweer and Johan Hachani, main contributors of this proteomics project and Rigas Karamanos for his critical reading. 


\section{References}

[1] Cecchelli, R., Berezowski, V., Lundquist, S., Culot, M., Renftel, M., Dehouck, M.-P. and Fenart, L. (2007) Modelling of the Blood-Brain Barrier in Drug Discovery and Development. Nature Reviews Drug Discovery, 6, 650-661. http://dx.doi.org/10.1038/nrd2368

[2] Abbott, N.J., Patabendige, A.A.K., Dolman, D.E.M., Yusof, S.R. and Begley, D.J. (2010) Structure and Function of the Blood-Brain Barrier. Neurobiology of Disease, 37, 13-25. http://dx.doi.org/10.1038/nrd2368

[3] Nag, S. (2011) Morphology and Properties of Brain Endothelial Cells. Methods in Molecular Biology, 686, 3-47. http://dx.doi.org/10.1007/978-1-60761-938-3_1

[4] Hawkins, B.T. and Davis, T.P. (2005) The Blood-Brain Barrier/Neurovascular Unit in Health and Disease. Pharmacological Reviews, 57, 173-185. http://dx.doi.org/10.1124/pr.57.2.4

[5] Pottiez, G., Flahaut, C., Cecchelli, R. and Karamanos, Y. (2009) Understanding the Blood-Brain Barrier Using Gene and Protein Expression Profiling Technologies. Brain Research Reviews, 62, 83-98. http://dx.doi.org/10.1016/j.brainresrev.2009.09.004

[6] Dehouck, M.P., Méresse, S., Delorme, P., Fruchart, J.C. and Cecchelli, R. (1990) An Easier, Reproducible, and Mass-Production Method to Study the Blood-Brain Barrier in Vitro. Journal of Neurochemistry, 54, 1798-1801. http://dx.doi.org/10.1111/j.1471-4159.1990.tb01236.x

[7] Dehouck, M.P., Jolliet-Riant, P., Brée, F., Fruchart, J.C., Cecchelli, R. and Tillement, J.P. (1992) Drug Transfer across the Blood-Brain Barrier: Correlation between in Vitro and in Vivo Models. Journal of Neurochemistry, 58, 1790-1797. http://dx.doi.org/10.1111/j.1471-4159.1992.tb10055.x

[8] Deracinois, B., Flahaut, C., Duban-Deweer, S. and Karamanos, Y. (2013) Comparative and Quantitative Global Proteomics Approaches: An Overview. Proteomes, 1, 180-218. http://dx.doi.org/10.3390/proteomes1030180

[9] Deracinois, B., Duban-Deweer, S., Pottiez, G., Cecchelli, R., Karamanos, Y. and Flahaut, C. (2012) TNAP and EHD1 Are Over-Expressed in Bovine Brain Capillary Endothelial Cells after the Re-Induction of Blood-Brain Barrier Properties. PLoS One, 7, e48428. http://dx.doi.org/10.1371/journal.pone.0048428

[10] Pottiez, G., Sevin, E., Cecchelli, R., Karamanos, Y. and Flahaut, C. (2009) Actin, Gelsolin and Filamin-A Are Dynamic Actors in the Cytoskeleton Remodelling Contributing to the Blood Brain Barrier Phenotype. Proteomics, 9, 1207-1219. http://dx.doi.org/10.1002/pmic.200800503

[11] Pottiez, G., Deracinois, B., Duban-Deweer, S., Cecchelli, R., Fenart, L., Karamanos, Y. and Flahaut, C. (2010) A Large-Scale Electrophoresis- and Chromatography-Based Determination of Gene Expression Profiles in Bovine Brain Capillary Endothelial Cells after the Re-Induction of Blood-Brain Barrier Properties. Proteome Science, 8, 57. http://dx.doi.org/10.1186/1477-5956-8-57

[12] Pottiez, G., Duban-Deweer, S., Deracinois, B., Gosselet, F., Camoin, L., Hachani, J., Couraud, P.-O., Cecchelli, R., Dehouck, M.-P., Fenart, L., Karamanos, Y. and Flahaut, C. (2011) A Differential Proteomic Approach Identifies Structural and Functional Components That Contribute to the Differentiation of Brain Capillary Endothelial Cells. Journal of Proteomics, 75, 628-641. http://dx.doi.org/10.1016/j.jprot.2011.09.002

[13] Méresse, S., Dehouck, M.P., Delorme, P., Bensaïd, M., Tauber, J.P., Delbart, C., Fruchart, J.C. and Cecchelli, R. (1989) Bovine Brain Endothelial Cells Express Tight Junctions and Monoamine Oxidase Activity in Long-Term Culture. Journal of Neurochemistry, 53, 1363-1371. http://dx.doi.org/10.1111/j.1471-4159.1989.tb08526.x

[14] Booher, J. and Sensenbrenner, M. (1972) Growth and Cultivation of Dissociated Neurons and Glial Cells from Embryonic Chick, Rat and Human Brain in Flask Cultures. Neurobiology, 2, 97-105.

[15] Culot, M., Lundquist, S., Vanuxeem, D., Nion, S., Landry, C., Delplace, Y., Dehouck, M.-P., Berezowski, V., Fenart, L. and Cecchelli, R. (2008) An in Vitro Blood-Brain Barrier Model for High Throughput (HTS) Toxicological Screening. Toxicology in Vitro, 22, 799-811. http://dx.doi.org/10.1016/j.tiv.2007.12.016

[16] Gosselet, F., Candela, P., Sevin, E., Berezowski, V., Cecchelli, R. and Fenart, L. (2009) Transcriptional Profiles of Receptors and Transporters Involved in Brain Cholesterol Homeostasis at the Blood-Brain Barrier: Use of an in Vitro Model. Brain Research, 1249, 34-42. http://dx.doi.org/10.1016/j.brainres.2008.10.036

[17] Duban-Deweer, S., Flahaut, C. and Karamanos, Y. (2012) The Proteome of Brain Capillary Endothelial Cells: Towards a Molecular Characterization of an in Vitro Blood-Brain Barrier Model. In: Karamanos, Y., Ed., Expression Profiling in Neuroscience (Vol. 64), Humana Press: Totowa, 161-179. http://dx.doi.org/10.1007/978-1-61779-448-3 10

[18] Peterson, G.L. (1977) A Simplification of the Protein Assay Method of Lowry et al. Which Is More Generally Applicable. Analytical Biochemistry, 83, 346-856. http://dx.doi.org/10.1016/0003-2697(77)90043-4

[19] Pont, F. and Fournié, J.J. (2010) Sorting Protein Lists with nwCompare: A Simple and Fast Algorithm for n-Way Comparison of Proteomic Data Files. Proteomics, 10, 1091-1094. http://dx.doi.org/10.1002/pmic.200900667 
[20] Huang, D.W., Sherman, B.T. and Lempicki, R.A. (2009) Systematic and Integrative Analysis of Large Gene Lists Using DAVID Bioinformatics Resources. Nature Protocols, 4, 44-57. http://dx.doi.org/10.1038/nprot.2008.211

[21] Mi, H., Dong, Q., Muruganujan, A., Gaudet, P., Lewis, S. and Thomas, P.D. (2010) PANTHER Version 7: Improved Phylogenetic Trees, Orthologs and Collaboration with the Gene Ontology Consortium. Nucleic Acids Research, 38 , D204-210. http://dx.doi.org/10.1093/nar/gkp1019

[22] Thomas, P.D., Campbell, M.J., Kejariwal, A., Mi, H., Karlak, B., Daverman, R., Diemer, K., Muruganujan, A. and Narechania, A. (2003) PANTHER: A Library of Protein Families and Subfamilies Indexed by Function. Genome Research, 13, 2129-2141. http://dx.doi.org/10.1101/gr.772403

[23] Deracinois, B., Pottiez, G., Chafey, P., Teerlink, T., Camoin, L., Davids, M., Broussard, C., Couraud, P.-O., Dehouck, M.-P., Cecchelli, R., Karamanos, Y. and Flahaut, C. (2013) Glial-Cell-Mediated Re-Induction of the Blood-Brain Barrier Phenotype in Brain Capillary Endothelial Cells: A Differential Gel Electrophoresis Study. Proteomics, 13, 11851199. http://dx.doi.org/10.1002/pmic.201200166

[24] Duban-Deweer, S., Hachani, J., Deracinois, B., Cecchelli, R., Flahaut, C. and Karamanos, Y. (2012) Proteomic Analysis of Plasma Membrane Proteins in an In Vitro Blood-Brain Barrier Model. In: Man, T.K., Ed., Proteomics-Human Diseases and Protein Functions, InTech, 391-416. http://dx.doi.org/10.5772/30543

[25] Hoshi, Y., Uchida, Y., Tachikawa, M., Inoue, T., Ohtsuki, S. and Terasaki, T. (2013) Quantitative Atlas of Blood-Brain Barrier Transporters, Receptors, and Tight Junction Proteins in Rats and Common Marmoset. Journal of Pharmaceutical Sciences, 102, 3343-3355. http://dx.doi.org/10.1002/jps.23575

[26] Uchida, Y., Tachikawa, M., Obuchi, W., Hoshi, Y., Tomioka, Y., Ohtsuki, S. and Terasaki, T. (2013) A Study Protocol for Quantitative Targeted Absolute Proteomics (QTAP) by LC-MS) MS: Application for Inter-Strain Differences in Protein Expression Levels of Transporters, Receptors, Claudin-5, and Marker Proteins at the Blood-Brain Barrier in ddY, FVB, and. Fluids Barriers CNS, 10, 21. http://dx.doi.org/10.1186/2045-8118-10-21 